\title{
Inhaltsverzeichnis des 4. Bandes
}

Busch, K.-Th., Individuelle architektonische Differenzen der Area striata 535

Haug, H., Über die Beziehungen des Hirngewichtes zum Grauzellkoeffizienten der Sehrinde bei den Primaten und einigen primitiven Säugern . . . . . . . . . . . . . . . . . . . . . . . . . . . 189

- und C. Kraus, Vergleichende Untersuchungen über die Cellodin- und Paraffineinbettung . . . . . . . . . . . . . . . . . 254

Hempel, K.-J., Histopathologische Untersuchungen am Supranucleus medialis-dorsalis thalami bei Schizophrenie . . . . . . . . . . . 205

- und M. Namba, Die Involution des Supranucleus medialis-dorsalis sowie der Lamella medialis und der Lamella interna thalami . . . . 43

- und W. M. Treff, Quantitativ-morphologische Untersuchungen am Supranucleus medialis-dorsalis thalami . . . . . . . . . . . . . 149

_ - Die Gliazelldichte bei klinisch Gesunden und Schizophrenen . . 371

- - Besteht eine Korrelation zwischen dem Nervenzellausfall und den Sohwundzellveränderungen bei der Katatonie? . . . . . . . . . . . 479

$\mathrm{Namba}, \mathrm{M}$., Über die feineren Strukturen des mediodorsalen Supranucleus und der Lamella medialis des Thalamus beim Menschen . . 1

Rabl, R., Strukturstudien an der Massa intermedia des Thalamus opticus 78

- Die pathocytologischen Veränderungen des Zentralnervensystems bei der Pfaundler-Hurlerschen Krankheit (Gargoylismus) . . . . . . . 455

Sanides, F., Vergleichend morphologische Untersuchungen an kleinen Nervenzellen und an Gliazellen . . . . . . . . . . . . . . . 113

- Vorläufige Darstellung eines histologischen Phänomens an cytoarchitektonischen Feldergrenzen. . . . . . . . . . . . . . . . 273

Schulze, A. F., Zur individuellen cytoarchitektonischen Gestaltung der linken und rechten Hemisphäre im Bereiche des Lobulus parietalis inferior . . . . . . . . . . . . . . . . . . . . 486

Treff, W. M. und K:-J. Hempel, Die Zelldichte bei Schizophrenen und klinisch Gesunden . . . . . . . . . . . . . . . . . 314

- - Quantitative Untersuchungen über relative Zell- und Zellkernvolumina bei klinisch Gesunden und Schizophrenen . . . . . . . . 412 